\section{Clinical Cases in Pediatric Dentistry}

Editor: Amr M. Moursi

Associate Editor: Amy L. Truesdale

Publisher:Wiley-Blackwell, Hoboken, NJ, USA

Language: English

ISBN: 978-1-119-29091-9

Edition: 2/e

Publish Year: 2020

Pages: 424, illustrated

Price: $€ 76.99$

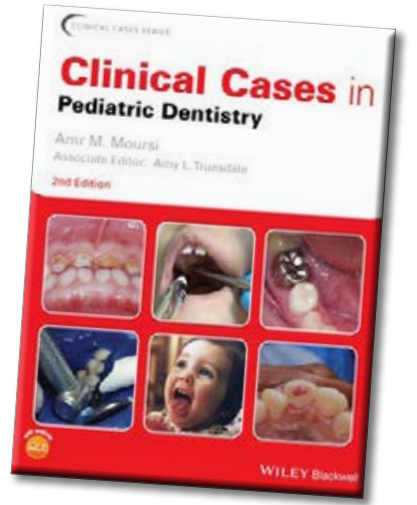

Iulia Ciolachi

DMD

Holistic Dental \& Medical Institute

of Bucharest-ROPOSTURO

Bucharest, Romania

e-mail:

driuliaciolachi@yahoo.ro

Clinical Cases in Pediatric Dentistry, $\mathbf{2}^{\text {nd }}$ Edition is aimed at pediatric dentists to help them have a holistic approach on treating infants. This book is also a source for dental teachers to expand their collection of clinical cases. The book is divided in nine chapters, accompanied by an index and a companion website.

The cases share the same structure, including medical and dental history, examination, assessment tools in infant oral cases, diagnosis, diagnostic tools, comprehensive treatment plan, prognosis, discussion, common complication and alternative treatment plans. Each case is accompanied by self-assessment questions with detailed answers and explanations.

Chapter 1, Early Childhood Oral Health, covers six cases and talks about: perinatal oral pathology, first dental visit, early childhood caries managed with silver diamine fluoride and interim therapeutic restorations and caries managed with general anesthesia.

Chapter 2, Restorative Dentistry, illustrates, in eight clinical cases, different classes of restorations either with glass ionomer or resin and strip crowns and zirconia crowns.

Chapter 3, Complex Pulp Therapy, approaches the complexed therapy in temporary and young permanent teeth through nine clinical cases.

Chapter 4, Orofacial Trauma, presents eight cases which exemplify the main dental trauma and how to treat it.

Chapter 5, Oral Medicine and Orofacial Pathology, illustrates seven different forms of oral pathology.

Chapter 6, Behavior Guidance and Medical Emergencies, presents seven cases which cover nonpharmacological and pharmacological behavior guidance, intraoperative pain management, nitrous oxide/oxygen sedation, airway management, allergic reactions and general anesthesia.

Chapter 7, Growth and Development, discusses, in seven cases, management of practical procedures.

Chapter 8, Medically Compromised Patients, presents the therapeutic approach for patients with general disorders such as congenital heart disease, cystic fibrosis, hemophilia A, acute lymphoblastic leukemia, liver transplant, type 1 diabetes, asthma, and Crohn's disease.

The last chapter, chapter 9, Patients with Disabilities, covers nine cases which explain the approach in Down syndrome, cerebral palsy, bronchopulmonary dysplasia, attention deficit hyperactivity disorder, seizure disorder, intellectual disability, autism spectrum disorder, sickle cell anemia, and intellectual disability.

This book is an essential working tool for dental students, postgraduate residents, and pediatric dentists preparing for board examinations and recertification. With a total of 66 cases, using a clear, concise, and consistent format, the book presents real-world cases that encompass all the important areas of pediatric dentistry.

DOI: http://www.stomaeduj.com 10.25241/stomaeduj.2020.7(1).bookreview.3

The Books Review is drafted in the reviewer's sole wording and illustrates his opinions. 